\title{
Effect of ROX Index on the Ventilator Free Days in High Flow Nasal Oxygen and Early Invasive Mechanical Ventilation in Adult COVID -19 Patients
}

\author{
Joslita Rebello $^{1}$, Monisha B. J. Neelankavil ${ }^{2}$, Ananth Srikrishna Somayaji ${ }^{3}$ \\ ${ }^{1}$ Post Graduate, Anaesthesiology, Father Muller Medical College Mangalore \\ ${ }^{2}$ Senior Resident, Anaesthesiology, Father Muller Medical College, Mangalore \\ ${ }^{3}$ Senior Resident, Father Muller Medical College, Mangalore \\ Corresponding Author: Joslita Rebello
}

\begin{abstract}
Background: High flow nasal oxygen (HFNO) is used as an alternative respiratory support in hypoxemic respiratory failure in COVID -19. However, the use of HFNO was associated with a lower risk of invasive mechanical ventilation and duration of stay in ICU. This study is aimed at comparing ventilator free days and duration of ICU stay between early mechanical ventilation and HFNO use in COVID -19 to predict the clinical outcome.

Methods: We performed a unicentre prospective observational analytical study on subjects with respiratory failure due to COVID -19 comparing effect of ROX index on ventilator free days with use of HFNO therapy and mechanical ventilation on first day of intensive care unit admission. Each group had 20 subjects. Clinical outcome was measured in terms of ventilator free days between two groups. Standard statistical comparisons were used to compare the length of ICU stay as secondary outcome. APACHE II and SOFA sores were compared and analysed between two groups.

Results: 40 adult subjects critically ill due to COVID -19 were included in the study with 20 in each group. Subjects in HFNO group had higher ventilator free days than those were put on early mechanical ventilation with significant difference. Whereas the duration of ICU stay was prolonged in HFNO group but there was no significant statistical difference.

Conclusions: In this prospective study HFNO had better clinical outcome in terms of ventilator free days compared to early mechanical ventilation. Duration of stay in ICU had no difference.
\end{abstract}

Keywords: Ventilator free days, HFNO, number of days in ICU, ROX index, APACHE II, SOFA score.

\section{INTRODUCTION}

High flow nasal oxygen (HFNO) is used as an alternative respiratory support in hypoxemic respiratory failure in COVID 19 patients. In patients with hypoxaemic respiratory failure, HFNO is more effective than conventional oxygen therapy in improving oxygenation. Patients with mildto-moderate hypoxaemic respiratory failure are most likely to benefit from HFNO ${ }^{(1)}$. Acute respiratory failure (ARF) caused by acute hypoxemia is the most common manifestation of severe coronavirus disease. COVID-19 patients are hospitalised in intensive care units may require invasive mechanical ventilation which is associated with a high mortality rate ${ }^{(2,3,4)}$. However, the use of HFNO was associated with a lower risk of invasive mechanical ventilation and duration of stay in ICU ${ }^{(5,6,7)}$. ROX index, defined as the ratio of oxygen saturation as measured by pulse oximetry 
$\left(\mathrm{Sp}_{\mathrm{O} 2}\right) / \mathrm{Fi}_{\mathrm{O} 2}$ to respiratory rate $(\mathrm{RR})$. The ROX index sensitive predictor of outcome of HFNO therapy in patients with ARF caused by pneumonia especially in covid 19. Studies regarding ROX index in predicting the outcome of use of invasive mechanical ventilation are less ${ }^{(8,9)}$. This study is aimed at analyzing the effect of ROX index at the time of ICU admission between early intubation and HFNO use on ventilator free days as the outcome.

Aim of the study is to analyse the effect of ROX index at the time of ICU admission in predicting the ventilator free days on high-flow nasal oxygen compared to early initiation of invasive mechanical ventilation, on adult patients with COVID19.

Objective of the study is to compare ventilator-free days as primary outcome and length of ICU stay as secondary outcome between early use of HFNO and early initiation of mechanical ventilation on adult patients with COVID-19.

\section{MATERIALS AND METHODS}

A prospective observational analytical study was conducted in Father muller medical college after obtaining ethical committee approval. A total of 40 subjects were included with 20 in each group. Age more than 18 years with confirmed nasopharyngeal sample for COVID -19, on HFNO or intubation on first day of ICU admission were included. Those subjects who were intubated outside ICU, on non invasive ventilation prior to HFNO with $\mathrm{GCS}<13$ and $\mathrm{Ph}<7.2$ were excluded from study.

After obtaining informed consent from patients and attenders those put on HFNO within first 24 hours of ICU admission were assigned to $\mathrm{H}$ group and patients put on invasive mechanical ventilator within first 24 hours of ICU admission were assigned to $\mathrm{V}$ group.

Patients were assigned to two groups based on inclusion and exclusion criteria. Data was collected by the personnel in ICU on the day of admission. Patient confidentiality maintained through the study. Demographic data such as Age, gender, weight, height, body mass index, number of comorbidities, day of illness on admission to ICU; vital signs such as heart rate systolic and diastolic blood pressure ,mean arterial pressure respiratory rate and severity assessment scales such as sequential organ failure assessment score, APACHE II score. The data was compared between HFNO and ventilator group.

Exposure of study was use of HFNO within 24 hours of ICU admission for initial oxygenation and comparing to the use of invasive mechanical ventilation within 24 hours of ICU admission. Patients initially receiving HFNO if switched within the 24hours window to invasive mechanical ventilation were assigned to $\mathrm{H}$ group. Deceased patients were considered to have zero ventilator free days. Number of ventilator free days was the primary outcome and calculated based on number of stay in ICU-number of days free from ventilator. Patient was followed until the discharge from intensive care unit. Secondary outcomes that is duration of stay in ICU was noted.

\section{Statistical analysis}

Statistical analysis done by Pearson Chi-square test and independent $t$ test to compare continuous variable. Software used was SPSS version 20.0.P value $<0.05$ was considered statistically significant.

\section{RESULTS}

This study was conducted in critically ill Covid -19 patients admitted in ICU in Father Muller Medical College and Hospital Mangalore after ethical committee approval. A total of 40 study subjects were assigned into two groups with 20 in each group based on inclusion and exclusion criteria by a trained anesthesiologist. Data required for the study was collected by the ICU personnel not involved in the study on the day of ICU admission. Demographic data and baseline parameters are shown in Table No 1. Primary outcome was to 
Joslita Rebello et.al. Effect of ROX index on the ventilator free days in high flow nasal oxygen and early invasive mechanical ventilation in adult COVID -19 patients.

compare the ventilator free days in in HFNO and early mechanical ventilation as initial oxygenation strategy. Secondary outcome was to compare the number of days stay in ICU between two groups. Increase in ventilator free days was associated with use of HFNO (mean of 7.1 days and standard deviation of 6.31 days) compared to early use of mechanical ventilation (mean of 1.1 days and standard deviation of 1.55 days) with significant statistical difference $(\mathrm{p}<0.001)$. No difference was observed in length ICU stay with use of HFNO (mean of 9.95 days and standard deviation of 6.21 days) compared to early use of mechanical ventilator (mean of 9.7 days and standard deviation of 4.05 days). Significant difference was observed in the ROX index between two groups $(\mathrm{p}<0.001)$.

Table No 1. Demographic data and Baseline parameters

\begin{tabular}{|c|c|c|c|c|c|}
\hline \multirow[b]{2}{*}{ PARAMETER } & \multicolumn{2}{|c|}{ Group H } & \multicolumn{2}{|c|}{ Group V } & \multirow[b]{2}{*}{ P value } \\
\hline & $\mathbf{N}$ & Mean \pm sd & $\mathbf{N}$ & Mean \pm sd & \\
\hline Age (years) & 20 & $52.55 \pm 16.42$ & 20 & $59.35 \pm 19.58$ & 0.241 \\
\hline Weight(Kg) & 20 & $70.4 \pm 9.42$ & 20 & $71.4 \pm 7.06$ & 0.706 \\
\hline Height(cms) & 20 & $166.5 \pm 6.7$ & 20 & $168.65 \pm 5.45$ & 0.273 \\
\hline $\mathrm{BMI}\left(\mathrm{kg} / \mathrm{m}^{2}\right)$ & 20 & $25.39 \pm 2.77$ & 20 & $25.16 \pm 2.13$ & 0.769 \\
\hline Number of comorbidities & 20 & $1.7 \pm 1.13$ & 20 & $2.4 \pm 1.27$ & 0.074 \\
\hline Day of illness on admission(days) & 20 & $6.8 \pm 1.88$ & 20 & $4.55 \pm 2.28$ & 0.002 \\
\hline HR(per minute) & 20 & $85.45 \pm 18.77$ & 20 & $97.2 \pm 18.28$ & 0.052 \\
\hline $\mathrm{SBP}(\mathrm{mmHg})$ & 20 & $131.55 \pm 15.76$ & 20 & $130.9 \pm 15.07$ & 0.895 \\
\hline DBP(mmHg) & 20 & $78.4 \pm 10.52$ & 20 & $77.85 \pm 12.54$ & 0.881 \\
\hline MAP(mmHg) & 20 & $93.85 \pm 10.86$ & 20 & $89.6 \pm 15.42$ & 0.32 \\
\hline RR(per minute) & 20 & $28.25 \pm 3.19$ & 20 & $30.35 \pm 2.72$ & 0.031 \\
\hline SPO2(\%) & 20 & $90.45 \pm 4.37$ & 20 & $87.1 \pm 3.39$ & 0.01 \\
\hline Temperature ${ }^{0} \mathrm{~F}$ & 20 & $99.07 \pm 0.8$ & 20 & $99.05 \pm 0.82$ & 0.94 \\
\hline $\mathrm{PH}$ & 20 & $7.44 \pm 0.05$ & 20 & $7.4 \pm 0.05$ & 0.025 \\
\hline $\mathrm{PaO} 2$ (mmHg) & 20 & $60 \pm 13.48$ & 20 & $57.4 \pm 12.27$ & 0.527 \\
\hline PCO2(mmHg) & 20 & $37.85 \pm 13.48$ & 20 & $34.45 \pm 6.76$ & 0.32 \\
\hline HCO3(mEq/L) & 20 & $24.86 \pm 3.54$ & 20 & $22.22 \pm 4.42$ & 0.044 \\
\hline FIO2 & 20 & $0.75 \pm 0.12$ & 20 & $0.83 \pm 0.1$ & 0.029 \\
\hline GCS & 20 & $14.95 \pm 0.22$ & 20 & $14.85 \pm 0.49$ & 0.411 \\
\hline SOFA SCORE & 20 & $4.8 \pm 1.06$ & 20 & $5.05 \pm 1.15$ & 0.478 \\
\hline APACHE II & 20 & $15.5 \pm 4.62$ & 20 & $19.95 \pm 5.43$ & 0.008 \\
\hline ROX INDEX & 20 & $4.44 \pm 0.92$ & 20 & $3.54 \pm 0.43$ & $<0.001$ \\
\hline NUMBER OF DAYS IN ICU & 20 & $9.95 \pm 6.21$ & 20 & $9.7 \pm 4.05$ & 0.881 \\
\hline VENTILATOR FREE DAYS & 20 & $7.1 \pm 6.31$ & 20 & $1.1 \pm 1.55$ & $<0.001$ \\
\hline
\end{tabular}

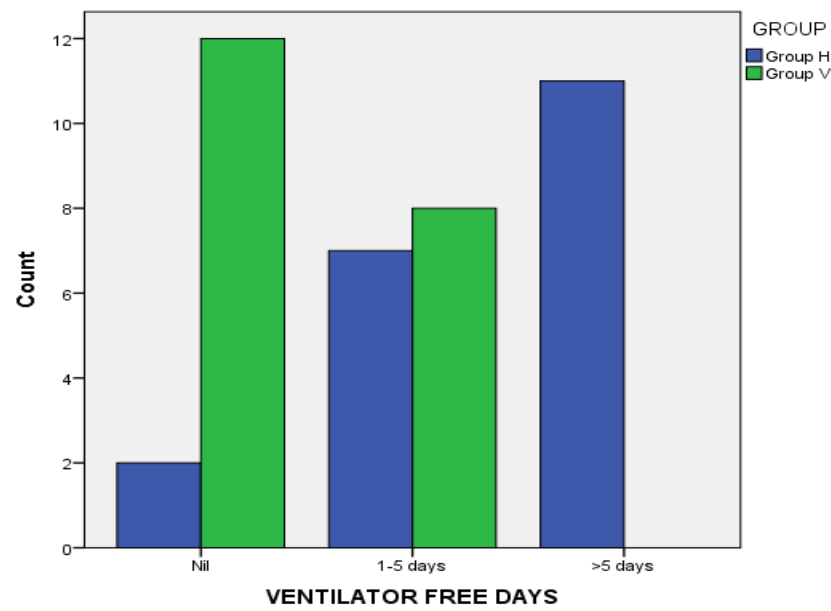

Figure 1: Graphical representation of ventilator free days between the two groups.

Figure 1 depicts the number of ventilator free days between the two groups. Ventilator free days are more in HFNO group. Out of 20 study subjects in more than $50 \%$ were free of ventilator for more than 5 days in HFNO group. Ventilator free days showed significant difference in HFNO group ( $<<0.001)$. 
Joslita Rebello et.al. Effect of ROX index on the ventilator free days in high flow nasal oxygen and early invasive mechanical ventilation in adult COVID -19 patients.

\section{DISCUSSION}

In this unicentre prospective observational analytical study involving critically ill COVID-19 patients with acute respiratory failure, ROX index was higher and associated with increased ventilator free days with HFNO use compared to early use of mechanical ventilator. No difference was observed in length of ICU stay.

Use of conservative non-invasive approach is superior to invasive approach in treating patients with significant hypoxia in patients with COVID -19 in spite of uncertainty and these findings are consistent with other studies ${ }^{(5,6)}$. ROX index has been used as a predictor of invasive mechanical ventilation requirement both in HFNO and NIV use ${ }^{(11)}$. ROX index has not been used as marker in predicting the outcomes in early use of invasive mechanical ventilation. Better clinical outcomes have lesser and economic use hospital resources. In addition to the ventilator free days and length of ICU stay this study provides information on demographic data, baseline parameters, SOFA and APACHE scoring. Demographic data (age, gender, weight, height, BMI) showed no statistical significance. Day of illness on admission to ICU was higher in HFNO group. APACHE II and SOFA scores were higher in ventilator group as they were sicker on admission. ROX index was higher in HFNO group and associated with increased ventilator free days during the ICU stay.

Following limitations of the study were observed. First, study was conducted in a single centre and comprises of small group. Second, the study results may vary with strains as the severity varies and was not taken into consideration in the study. Third, length of ICU stay was more in HFNO group as subjects in HFNO group were put on conventional oxygen therapy following discontinuation of HFNO, deceased were more in ventilator group reducing the overall ICU stay.

\section{CONCLUSION}

In this observational study, conducted in 40 adult critically ill COVID19 patients with acute respiratory failure admitted to ICU, use of HFNO was associated with increased ventilator free days compared to use of invasive mechanical ventilation. Higher ROX index at admission to ICU is associated with increased ventilator free days. Further studies are required to confirm the outcome using ROX index in early use of HFNO or invasive mechanical ventilation

\section{Acknowledgement: None}

\section{Conflict of Interest: None}

\section{Source of Funding: None}

\section{Ethical Approval: Approved}

\section{REFERENCES}

1. Renda T, Corrado A, Iskandar G, Pelaia G, Abdalla K, Navalesi P. High-flow nasal oxygen therapy in intensive care and anaesthesia. British Journal of Anaesthesia. 2018;120(1):18-27.

2. Wang D, Hu B, Hu C, Zhu F, Liu X, Zhang $\mathrm{J}$ et al. Clinical Characteristics of 138 Hospitalized Patients With 2019 Novel Coronavirus-Infected Pneumonia in Wuhan, China. JAMA. 2020;323(11):1061.

3. Xie J, Wu W, Li S, Hu Y, Hu M, Li J et al. Clinical characteristics and outcomes of critically ill patients with novel coronavirus infectious disease (COVID-19) in China: a retrospective multicenter study. Intensive Care Medicine. 2020;46(10):1863-1872.

4. Ince U, Duran H, Yildirim Y, Gokce S. The Effect of Co-Morbid Diseases on The Need for Mechanical Ventilation and Mortality of Patients with Covid-19 in Intensive Care Unit. International Medical Journal. 2021;10(3):684.

5. Bonnet N, Martin O, Boubaya M, Levy V, Ebstein N, Karoubi P et al. High flow nasal oxygen therapy to avoid invasive mechanical ventilation in SARS-CoV-2 pneumonia: a retrospective study. Annals of Intensive Care. 2021;11(1).

6. Ouyang L, Yu M, Zhu Y, Gong J. Respiratory supports of COVID-19 patients 
Joslita Rebello et.al. Effect of ROX index on the ventilator free days in high flow nasal oxygen and early invasive mechanical ventilation in adult COVID -19 patients.

in intensive care unit: A systematic review. Heliyon. 2021;7(4):e06813.

7. Lyons C, Callaghan $M$. The use of high-flow nasal oxygen in COVID -19. Association of Anaesthetists. 2020;75(7): 843-47.

8. Hill N, Ruthazer R. Predicting Outcomes of High-Flow Nasal Cannula for Acute Respiratory Distress Syndrome. An Index that ROX. American Journal of Respiratory and Critical Care Medicine. 2019;199(11): 1300-1302.

9. 9.Mellado-Artigas, R., Mujica, L.E., Ruiz, M.L. et al. Predictors of failure with highflow nasal oxygen therapy in COVID-19 patients with acute respiratory failure: a multicenter observational study. $j$ intensive care 9, 23 (2021).
10. Mellado-Artigas R, Ferreyro B, Angriman F, Hernández-Sanz M, Arruti E, Torres A et al. High-flow nasal oxygen in patients with COVID-19-associated acute respiratory failure. Critical Care. 2021;25(1).

11. Mukhtar A, Rady A, Hasanin A, Lotfy A, El Adawy A, Hussein A et al. Admission SpO2 and ROX index predict outcome in patients with COVID-19. The American Journal of Emergency Medicine. 2021;50:106-110.

How to cite this article: Rebello J, Monisha B. J. Neelankavil, Somayaji AS. Effect of ROX index on the ventilator free days in high flow nasal oxygen and early invasive mechanical ventilation in adult COVID-19 patients. Int $J$ Health Sci Res. 2021; 11(11): 203-207. DOI: https://doi.org/10.52403/ijhsr.20211126 